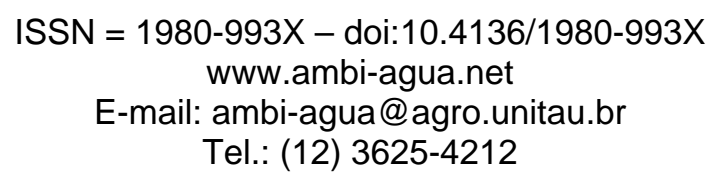

\title{
Estimativa do fator topográfico da USLE a partir de três algoritmos
} (doi:10.4136/ambi-agua.149)

\author{
Paulo Tarso Sanches de Oliveira ${ }^{1}$; Dulce Buchala Bicca Rodrigues ${ }^{1}$; Teodorico Alves \\ Sobrinho $^{1}$; Elói Panachuki ${ }^{2}$ \\ ${ }^{1}$ Universidade Federal de Mato Grosso do Sul - UFMS \\ E-mail: paulotarsoms@gmail.com; dulcebbr@gmail.com; talves@ufms.br \\ ${ }^{2}$ Universidade Estadual de Mato Grosso do Sul - UEMS \\ E-mail: eloip@uems.br
}

\section{RESUMO}

Diversos pesquisadores têm buscado modelar por meio de equações matemáticas o fator topográfico (comprimento de rampa e declividade) da Universal Soil Loss Equation (USLE) em diferentes condições de relevo. No presente trabalho, analisou-se o desempenho de três equações na geração do fator topográfico bem como a influência da declividade no seu cálculo. Dados topográficos processados em sistema de informação geográfica foram utilizados na obtenção do modelo digital de elevação. Esse foi considerado para o cálculo do fator topográfico no software USLE-2D. Verificou-se que existem diferenças entre os resultados obtidos por diferentes algoritmos e que áreas com declives acentuados proporcionam diferenças maiores. Nas equações estudadas, a declividade correspondeu a $75,1 \%, 83,8 \%$ e $82,8 \%$ do fator topográfico, portanto a declividade possui maior influência que o comprimento de rampa no cálculo do fator topográfico. A escolha da equação a ser aplicada para obtenção do fator topográfico deve obedecer às peculiaridades do relevo local.

Palavras-chave: modelo digital de elevação; comprimento de rampa; erosão hídrica.

\section{Estimating of the USLE topographic factor using three algorithms}

\section{ABSTRACT}

Several researchers have proposed the mathematical modeling of the topographic factor (slope length and slope steepness) of the Universal Soil Loss Equation (USLE) in different conditions of relief. This paper presents an analysis of the performance of three equations in the production of the topographic factor as well as the influence of slope steepness in its estimation. Topographic data processed in a geographic information system were used to derive the digital elevation model that was applied to calculate the topographic factor using the software USLE-2D. Differences between the results when using distinct algorithms were found and areas with larger slope steepness resulted in greater differences. The slope corresponded to $75.1 \%, 83.8 \%$ and $82.8 \%$ of the topographic factor in the studied equations. Therefore, the slope has more influence than the length of the ramp in the calculation of the topographic factor. In addition, the choice of the equation to be applied to obtain such factor is dependent upon the local relief.

Keywords: digital elevation model; slope length; water erosion.

\section{INTRODUÇÃO}

No desenvolvimento de estudos da variação espacial e temporal da erosão hídrica do solo são utilizadas técnicas de modelagem que proporcionam a avaliação de impactos na agricultura, solos e recursos hídricos (Renschler e Harbor, 2002), fornecendo informações úteis na tomada de decisão no planejamento conservacionista do solo e água (Schiettecatte et 
al., 2008). Dentre os modelos empíricos de predição da erosão, pode-se destacar a Universal Soil Loss Equation (USLE) proposta por Wischmeier e Smith (1978).

A USLE é composta por seis fatores que resultam na estimativa da perda de solo média anual, sendo: erosividade (R); erodibilidade (K); comprimento de rampa (L); declividade (S); uso e manejo do solo (C); e, práticas conservacionistas (P). Todos esses fatores devem ser estudados de forma individual e local antes da aplicação do modelo (Onyando et al., 2005; Irvem et al., 2007). A associação dos fatores $\mathrm{L}$ e $\mathrm{S}$ é denominada fator topográfico (Wischmeier e Smith, 1978).

Para uma mesma categoria de uso do solo (fatores C e P constantes), o fator topográfico (LS) proporciona maior influência na variação da perda de solo (Weill e Sparovek, 2008). O aumento do fator topográfico pode produzir maior velocidade de escoamento superficial e erosão. Além disso, a perda de solo é consideravelmente mais sensível às mudanças de declividade do que a mudanças no comprimento de rampa (McCool et al., 1987; Van Remortel et al., 2004).

A obtenção dos fatores L e S em bacias hidrográficas apresenta dificuldades em virtude do declive, que pode ser reto, côncavo, convexo ou uma combinação de formatos. Diversos pesquisadores buscaram descrever por meio de equações matemáticas o comportamento desses fatores em condições distintas. Wischmeier e Smith (1978) desenvolveram estudos para declives irregulares, atribuindo pesos conforme as características dos trechos de declive, côncava ou convexa. McCool et al. $(1987,1989)$ propuseram o cálculo dos fatores L e S em função da relação da erosão entressulcos e em sulcos e para valores de declividade superiores a 9\%. Liu et al. (1994) desenvolveram pesquisas nas regiões montanhosas da China e propuseram, para situações de declividades elevadas, modificações nas equações de McCool et al. (1987, 1989). Nearing (1997) propôs uma equação que engloba os fatores de declividades elevadas de McCool et al. $(1987,1989)$ e a equação para vertentes com declives mais íngremes de Liu et al. (1994).

Desse modo, o objetivo da presente pesquisa foi analisar os desempenhos das equações de Wischmeier e Smith (1978), de McCool et al. (1987, 1989) e de Nearing (1997) na geração do fator topográfico, bem como avaliar a influência da declividade no cálculo desse fator.

\section{MATERIAL E MÉTODOS}

\subsection{Delineamento geral do estudo}

O estudo foi realizado tomando-se por base a bacia hidrográfica do Ribeirão Salobra, com área aproximada de $540 \mathrm{~km}^{2}$, que está localizada entre as coordenadas de latitudes $20^{\circ}$ $12^{\prime} \mathrm{S}$ a $20^{\circ} 28^{\prime} \mathrm{S}$ e longitudes $54^{\circ} 55^{\prime} \mathrm{W}$ a $55^{\circ} 16^{\prime} \mathrm{W}$, com altitudes variando de 200 a 400 metros e declividade média de $2 \%$ (Figura 1 ).

A análise da influência da declividade no cálculo do fator topográfico foi realizada por meio de três equações, aplicadas no software USLE-2D (Desmet e Govers, 1996), tomando por base o Modelo Digital de Elevação (MDE). Em seguida, foram verificadas as relações entre as equações e a conformidade do relevo, que propiciaram as variações nos valores de LS (Figura 2). 


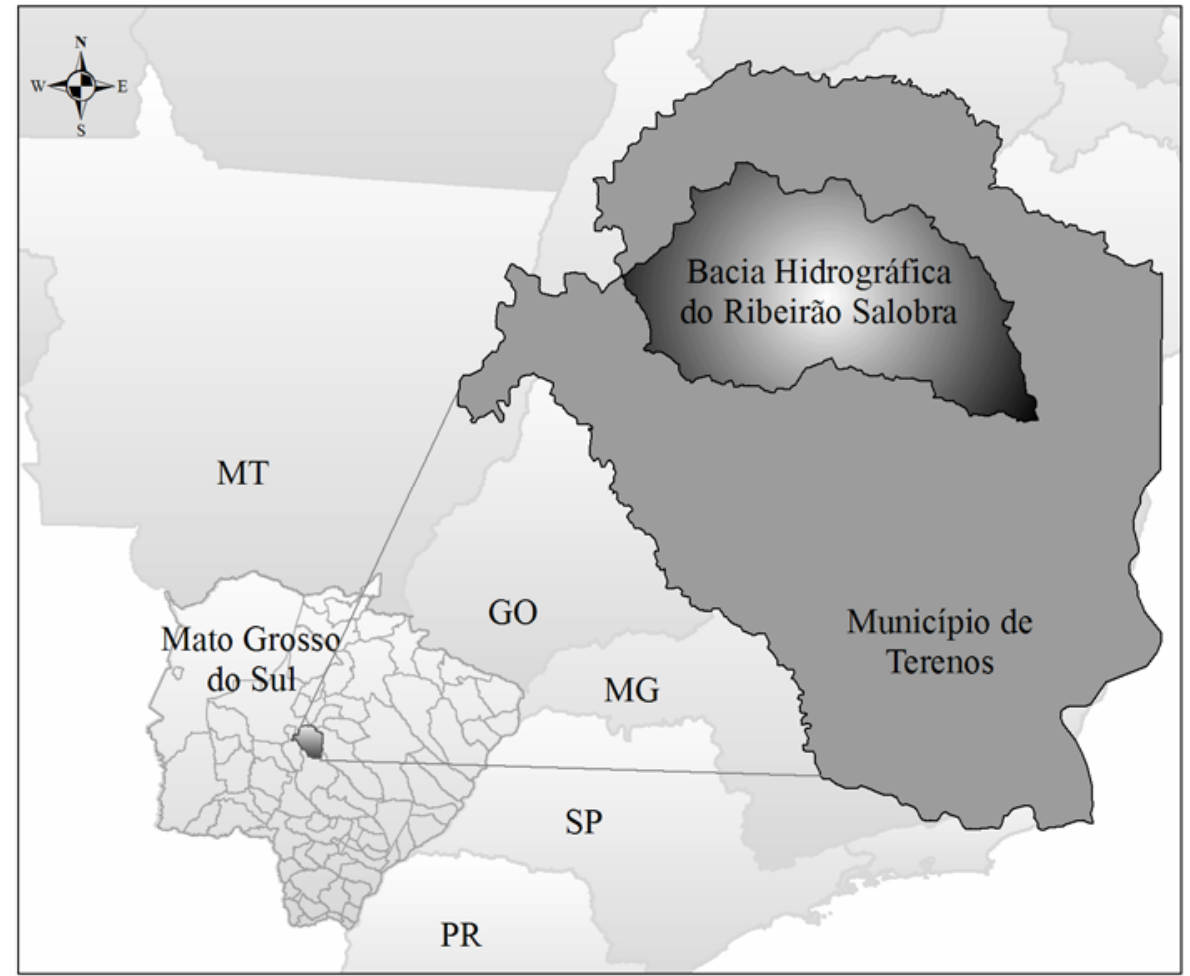

Figura 1. Localização da área em estudo.

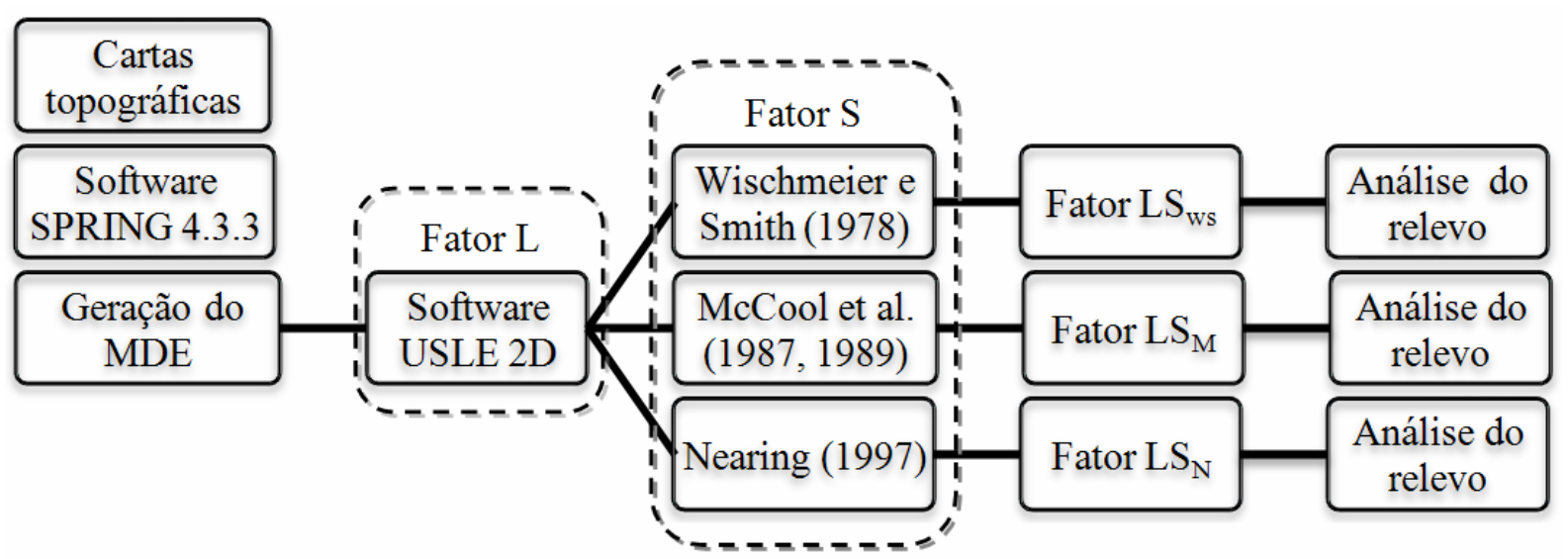

Figura 2. Esquema geral do cálculo do fator topográfico (LS), por três métodos distintos.

\subsection{Obtenção do Modelo Digital de Elevação (MDE)}

Para obtenção do MDE, foi efetuada a vetorialização da base topográfica, folhas Campo Grande SF. 21-X-B-II (DSG, 1979) e Palmeiras SF. 21-X-B-I (DSG, 1988), ambas na escala 1:100.000, representadas por curvas de nível equidistantes $40 \mathrm{~m}$, pontos cotados e hidrografia. Então, obteve-se o MDE a partir da transformação da grade triangular em retangular de $30 \mathrm{x}$ 30 metros, utilizando-se para tanto o aplicativo SPRING 4.3.3 (Câmara et al., 1996), do Sistema de Informação Geográfica (SIG).

\subsection{Obtenção do Fator $L$}

O MDE gerado foi processado no software USLE-2D para o cálculo do fator topográfico. O fator L foi obtido por meio do cálculo que considera a área de contribuição superficial por unidade de largura. Assim, é calculado por meio da divisão da área de contribuição pela largura ao longo do fluxo que pode passar dentro de uma célula. Essa largura depende da direção do fluxo e é calculada utilizando o aspecto de direção (Desmet e Govers, 1996) (Equação 1). 
OLIVEIRA, P. T. S.; RODRIGUES, D. B. B.; ALVES SOBRINHO, T.; PANACHUKI, E. Estimativa do fator topográfico da USLE a partir de três algoritmos. Ambi-Agua, Taubaté, v. 5, n. 2, p. 217-225, 2010. (doi:10.4136/ambi-agua.149)

$L_{i, j}=\left[\left(A_{i, j}+D^{2}\right)^{m+1}-\left(A_{i, j}\right)^{m+1}\right] /\left[x_{i, j}{ }^{m} \cdot D^{m+2} \cdot(22,13)^{m}\right]$

em que: $L_{i, j}=$ fator de comprimento de vertente de uma célula com coordenadas $(i, j) ; A_{i, j}=$ área de contribuição de uma célula com coordenadas $(i, j)\left(\mathrm{m}^{2}\right) ; \mathrm{D}=$ tamanho da grade de células $(\mathrm{m}) ; \mathrm{x}_{\mathrm{i}, \mathrm{j}}=$ valor da direção do fluxo; e, m: coeficiente dependente da declividade.

O software USLE-2D disponibiliza quatro algoritmos para elaboração do fator S, dentre os quais foram utilizados os seguintes: Wischmeier e Smith (1978), McCool et al. (1987, 1989) e Nearing (1997).

\subsection{Obtenção do Fator S}

\subsubsection{Algoritmo de Wischmeier e Smith (1978)}

O expoente (m) da Equação 1 foi calculado de acordo com Wischmeier e Smith (1978), sendo: $\mathrm{S}<1 \% \mathrm{~m}=0,2 ; 1 \% \leq \mathrm{S} \leq 3 \% \mathrm{~m}=0,3 ; 3 \%<\mathrm{S} \leq 5 \% \mathrm{~m}=0,4 ; \mathrm{e}, \mathrm{S}>5 \% \mathrm{~m}=0,5$. Utilizou-se a Equação 2 proposta por Wischmeier e Smith (1978) para o cálculo do fator S.

$\mathrm{S}_{\mathrm{ws}}=65,41 \operatorname{sen}^{2} \theta+4,56 \operatorname{sen} \theta+0,065$

sendo: $\theta=$ ângulo em graus da encosta.

\subsubsection{Algoritmo de McCool et al. $(1987,1989)$}

Aplicou-se a Equação 3 proposta por McCool et al. (1987, 1989), que também é utilizada na Revised Universal Soil Loss Equation (RUSLE) (Renard et al., 1997). Os valores do expoente (m) da Equação 1 são calculados conforme Equação 3.

$m=\beta /(1+\beta)$

Sendo $\beta$ o quociente entre a erosão em sulcos e entre sulcos podendo ser calculado conforme Equação 4 (McCool et al., 1989).

$\beta=(\operatorname{sen} \theta / 0,0896) /\left[3(\operatorname{sen} \theta)^{0,8}+0,56\right]$

A inclinação do declive (S) foi calculada de acordo com McCool et al. (1987) (Equações 5 e 6$)$.

$\mathrm{S}_{\mathrm{M}}=10,8 \operatorname{sen} \theta+0,03(\mathrm{~S}<9 \%)$

$\mathrm{S}_{\mathrm{M}}=16,8 \operatorname{sen} \theta-0,50(\mathrm{~S} \geq 9 \%)$

em que: $\theta=$ ângulo em graus da encosta.

\subsubsection{Algoritmo de Nearing (1997)}

Utilizou-se a Equação 7 proposta por Nearing (1997) no cálculo de fator S juntamente com a metodologia de McCool et al. (1989) para o cálculo do expoente m da Equação 1.

$\mathrm{S}_{\mathrm{N}}=1,5+17 / 1+\mathrm{e}^{(2,3-6,1 \operatorname{sen} \theta)}$

em que: $\theta=$ ângulo da encosta (graus). 
OLIVEIRA, P. T. S.; RODRIGUES, D. B. B.; ALVES SOBRINHO, T.; PANACHUKI, E. Estimativa do fator topográfico da USLE a partir de três algoritmos. Ambi-Agua, Taubaté, v. 5, n. 2, p. 217-225, 2010. (doi:10.4136/ambi-agua.149)

\subsection{Análise dos dados}

Analisou-se a diferença entre os fatores topográficos resultantes da aplicação das 3 equações acima referidas, por meio de análise estatística realizada pelo método de KruskalWallis (Kruskal e Wallis, 1952), sob um intervalo de confiança de 95\%.

Os fatores LS obtidos foram correlacionados com declividade presente em cada unidade espacial. Para tanto, o software IDRISI (Eastman, 2003) foi utilizado na obtenção da declividade a partir do MDE (Figura 3).

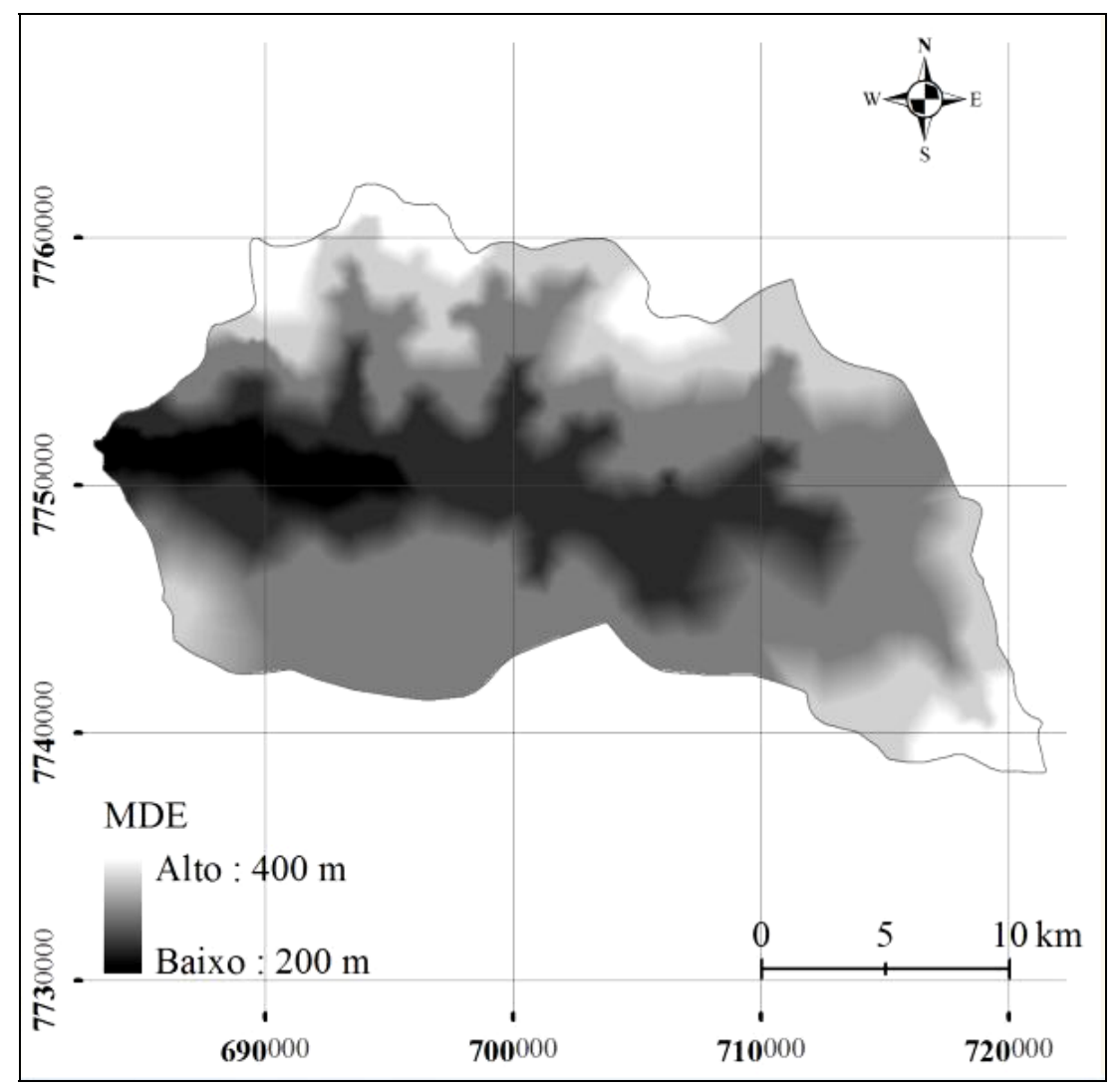

Figura 3. Modelo Digital de Elevação da área em estudo.

A correlação dos dados foi obtida a partir da função Regress do software IDRISI, sendo analisado o coeficiente de correlação $(r)$, o coeficiente de determinação $\left(r^{2}\right)$ e significância (teste $\mathrm{t}$, para $\alpha=0,1$ ). Nas três equações estudadas, verificou-se correlação linear entre as variáveis.

\section{RESULTADOS E DISCUSSÃO}

Os resultados apresentam variabilidade conforme observado nos valores de desvio padrão, coeficiente de variação e variância da amostra (Tabela 1). Isso em virtude da variação dos valores de $\mathrm{L}$ e $\mathrm{S}$ dentro de uma unidade complexa como uma bacia hidrográfica.

Tabela 1. Estatísticas descritivas do fator topográfico (LS), quando obtido por três diferentes algoritmos.

\begin{tabular}{l|c|c|c}
\hline Estatística Descritiva & Wischmeier e Smith (1978) & McCool et al. (1987, 1989) & Nearing (1997) \\
\hline Média & 0,677 & 0,739 & 0,703 \\
Mediana & 0,191 & 0,120 & 0,129 \\
Desvio padrão & 0,974 & 1,025 & 0,968 \\
Coeficiente de variação & 1,288 & 1,195 & 1,206 \\
Variância da amostra & 0,949 & 1,052 & 0,936 \\
\hline
\end{tabular}


Verifica-se o comportamento distinto da ocorrência do fator topográfico na bacia hidrográfica em estudo, referente a cada equação aplicada. As posições dos quartis evidenciam essa variação (Figura 4).

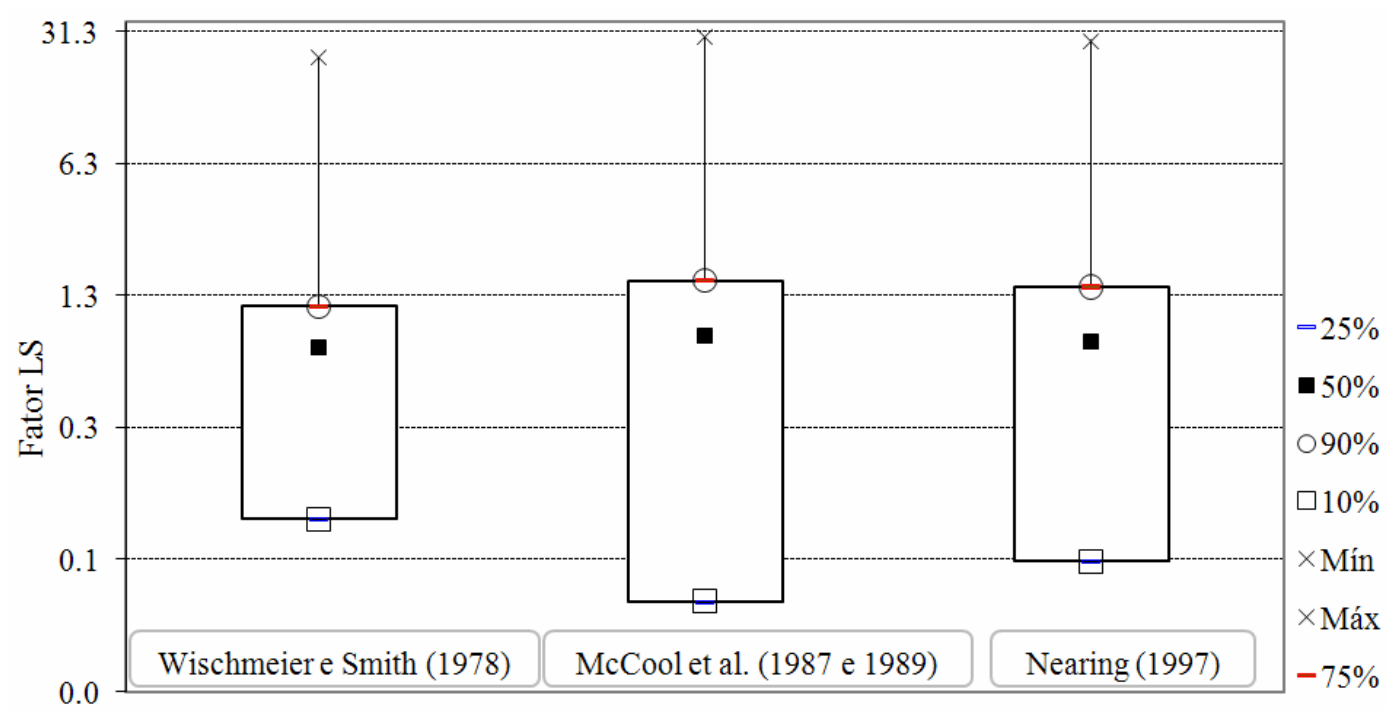

Figura 4. Box plot para valores calculados do fator topográfico.

É possível verificar maior semelhança entre os resultados obtidos a partir da equação McCool et al. (1987, 1989) e Nearing (1997). Entretanto, verificou-se que existe diferença estatística $(p<0,05)$ entre os resultados obtidos a partir das três equações.

Nota-se que, em áreas de baixa declividade, os valores do fator topográfico apresentamse semelhantes. Áreas com declive acentuado proporcionam maiores diferenças entre os resultados (Figura 5). Renard et al. (1997) demonstram que em encostas menos íngremes, o fator topográfico calculado pelas equações de McCool et al. (1987, 1989) apresenta-se próximo dos valores estabelecidos pelo método de Wischmeier e Smith (1978). No entanto, em condições de declividades mais acentuadas, os valores de LS determinados por McCool et al. $(1987,1989)$ são equivalentes à metade do valores de LS obtidos na USLE.

O valor médio do fator topográfico obtido na bacia hidrográfica em estudo é considerado baixo. No entanto, existem áreas com elevados valores, podendo ocasionar assim, maior escoamento superficial resultando em condições favoráveis à erosão hídrica. Essas áreas estão concentradas na região norte da bacia hidrográfica estudada (Figura 5).

Foram obtidos diferentes níveis de correlação entre o fator topográfico e a declividade para cada equação estudada. Em ordem decrescente, as equações de McCool et al. $(1987,1989)$, Nearing (1997) e Wischmeier e Smith (1978) possibilitaram maiores valores de correlação (Figura 6). 
OLIVEIRA, P. T. S.; RODRIGUES, D. B. B.; ALVES SOBRINHO, T.; PANACHUKI, E. Estimativa do fator topográfico da USLE a partir de três algoritmos. Ambi-Agua, Taubaté, v. 5, n. 2, p. 217-225, 2010. (doi:10.4136/ambi-agua.149)

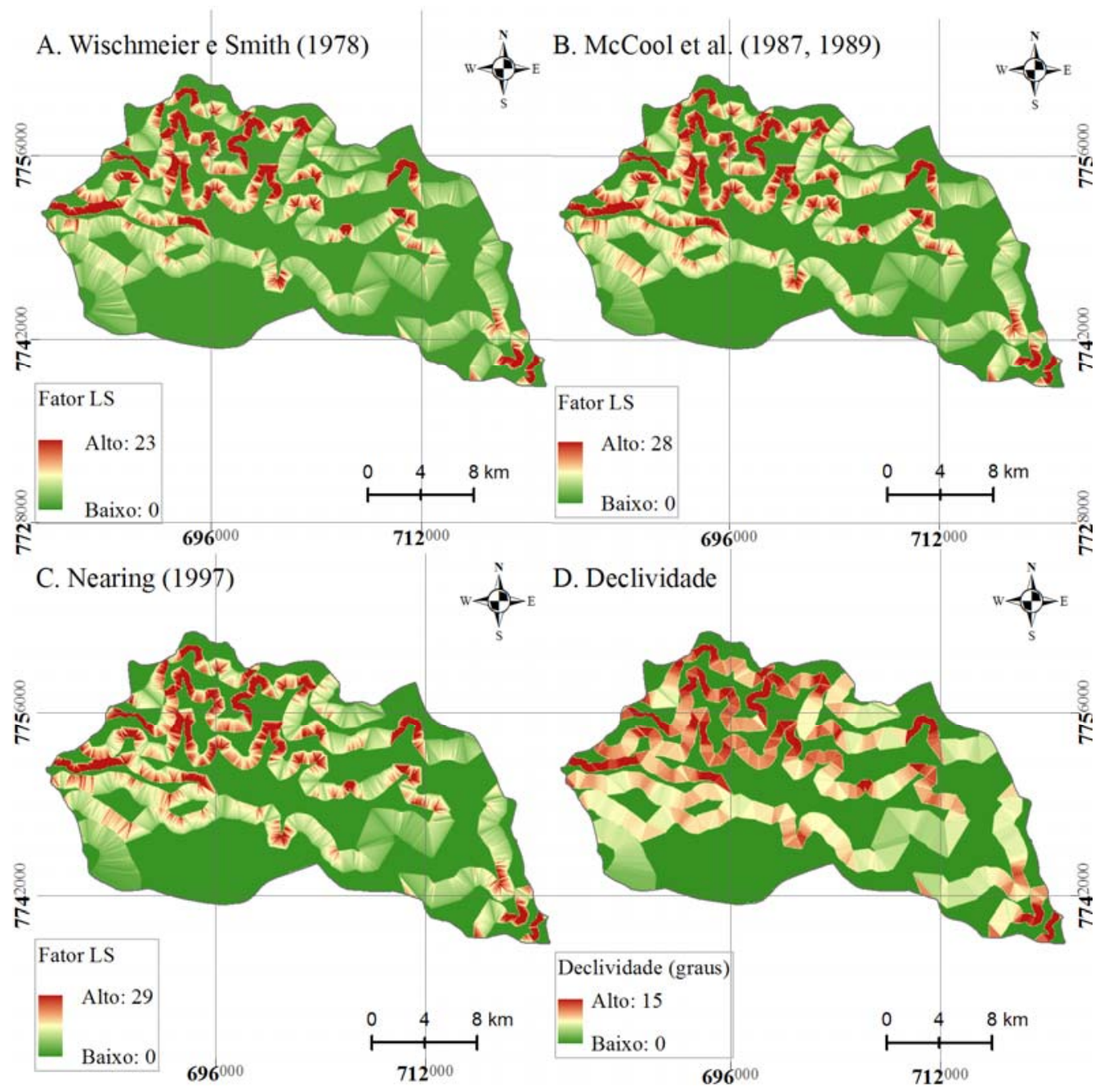

Figura 5. Mapas dos fatores topográficos (LS) quando obtidos por três diferentes equações (A, B e C) e da declividade (D).
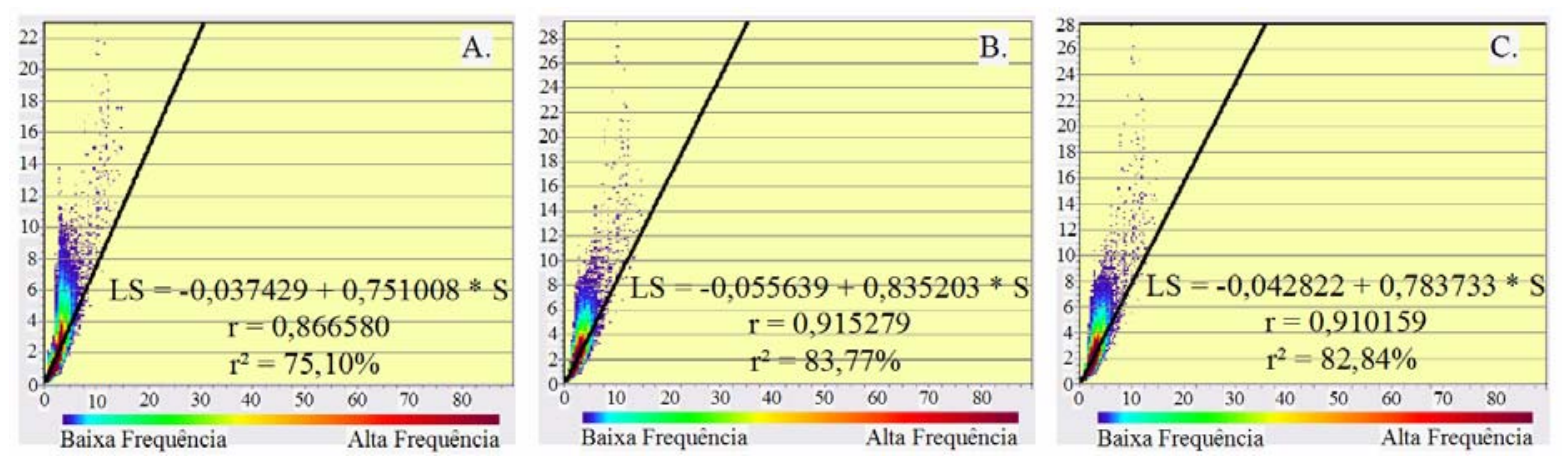

Figura 6. Correlação entre a declividade (eixo x) e o fator topográfico (eixo y). A. Wischmeier e Smith (1978); B. McCool et al. (1987, 1989); C. Nearing (1997).

Em virtude das equações de McCool et al. (1987,1989) e Nearing (1997) contemplarem valores de declividade superiores a $9 \%$, ao contrário da equação de Wischmeier e Smith (1978), as primeiras apresentaram melhores resultados de correlação com a declividade (Figura 6).

Considerando o valor obtido do coeficiente de determinação, as equações de McCool et al. (1987, 1989), Nearing (1997) e Wischmeier e Smith (1978) indicam que a declividade abrange $83,77 \%, 82,84 \%$ e $75,10 \%$ do fator topográfico, respectivamente. Portanto, a 
OLIVEIRA, P. T. S.; RODRIGUES, D. B. B.; ALVES SOBRINHO, T.; PANACHUKI, E. Estimativa do fator topográfico da USLE a partir de três algoritmos. Ambi-Agua, Taubaté, v. 5, n. 2, p. 217-225, 2010. (doi:10.4136/ambi-agua.149)

declividade possui maior influência que o comprimento de rampa no cálculo do fator topográfico.

A partir de valores de declividade aplicados a qualquer uma das três equações de correlação gerada neste estudo, pode-se chegar a uma aproximação do fator topográfico com boa precisão para a área em estudo.

\section{CONCLUSÕES}

A declividade possui maior influência que o comprimento de rampa no cálculo do fator topográfico.

Existem diferenças entre os resultados obtidos do fator topográfico (LS) pelos três algoritmos utilizados. Áreas com declives acentuados proporcionam maiores diferenças.

Em regiões com declividade acentuada, superior a 9\%, recomenda-se utilizar a equação de McCool et al. (1987, 1989) ou Nearing (1997).

A escolha da equação a ser aplicada para obtenção do fator topográfico deve obedecer às peculiaridades de cada equação em relação ao relevo local.

\section{AGRADECIMENTOS}

Os autores expressam seus agradecimentos ao Conselho Nacional de Desenvolvimento Científico e Tecnológico - CNPq e à Fundação de Apoio ao Desenvolvimento do Ensino, Ciência e Tecnologia do Estado de Mato Grosso do Sul - FUNDECT, pelo auxílio financeiro concedido para a realização do trabalho.

\section{REFERÊNCIAS}

CÂMARA, G.; SOUZA, R. C. M.; FREITAS, U. M.; GARRIDO, J. SPRING: Integrating remote sensing and GIS by object-oriented data modelling. Computers \& Graphics, v. 20, n. 3, p. 395-403, 1996.

DESMET, P. J. J; GOVERS, G. A GIS-procedure for automatically calculating the USLE LSfactor on topographically complex landscape units. Journal of Soil and Water Conservation, v. 51, n. 5, p. 427-433, 1996.

DIRETORIA DO SERVIÇO GEOGRÁFICO BRASILEIRO - DSG. Carta Palmeiras. Folha SF. 21. X. B. III. Escala 1:100.000. Ministério do Exército. Região Centro-Oeste do Brasil. Brasília: MEX, 1988.

DIRETORIA DO SERVIÇO GEOGRÁFICO BRASILEIRO - DSG. Carta Campo Grande. Folha SF. 21-X-B-II. Escala 1:100.000. Ministério do Exército. Região Centro-Oeste do Brasil. Brasília: MEX, 1979.

EASTMAN, J. R. Idrisi GIS software - user's manual. Worcester: Clark University, 2003.

IRVEM, A.; TOPALOGLU, F.; UYGUR, V. Estimating spatial distribution of soil loss over Seyhan River Basin in Turkey. Journal of Hydrology, v. 336, p. 30-37, 2007.

KRUSKAL, W. H.; WALLIS, W. A. Use of ranks in one-criterion variance analysis. Journal of the American Statistical Association, v. 47, n. 260, p. 583-621, 1952.

LIU, B. Y.; NEARING, M. A.; RISSE, L. M. Slope gradient effects on soil loss for steep slopes. Transactions of the American Society of Agricultural Engineers, v. 37, p. 1835-1840, 1994. 
MCCOOL, D. K.; BROWN, L. C.; FOSTER, G. R. Revised slope steepness factor for the Universal Soil Loss Equation. Transactions of the American Society of Agricultural Engineers, v. 30, p. 1387-1396, 1987.

MCCOOL, D. K.; FOSTER, G. R.; MUTCHLER, C. K.; MEYER, L. D. Revised slope length factor for the Universal Soil Loss Equation, Transactions of the American Society of Agricultural Engineers, v. 32, p. 1571-1576, 1989.

NEARING, M. A. A single, continuous function for slope steepness influence on soil loss. Soil Science Society of America Journal, v. 61, n. 3, p. 917-919, 1997.

ONYANDO, J. O.; KISOYAN, P.; CHEMELIL, M. C. Estimation of potential soil erosion for River Perkerra Catchment in Kenya. Water Resources Management, v. 19, p. 133$143,2005$.

RENARD, K. G.; FOSTER, G. R.; WEESIES, G. A.; MCCOOL, D. K.; YODER, D. C. Predicting soil erosion by water: a guide to conservation planning with the revised universal soil loss equation (RUSLE). Washington: USDA Agriculture Handbook, 1997.

RENSCHLER, C. S.; HARBOR, J. Soil erosion assessment tools from point to regional scales-the role of geomorphologists in land management research and implementation. Geomorphology, v. 47, p. 189-209, 2002.

SCHIETTECATTE, W.; D'HONDT, L.; CORNELIS, W. M.; ACOSTA, M. L.; LEAL, Z.; LAUWERS, N. et al. Influence of landuse on soil erosion risk in the Cuyaguateje watershed (Cuba). Catena, v. 74, p. 1-12, 2008.

VAN REMORTEL, R. D.; MAICHLE, R. W.; HICKEY, R. J. Computing the ls factor for the revised universal soil loss equation through array-basedslope processing of digital elevation data using a $\mathrm{C}++$ executable. Computers \& Geosciences, v. 30, p. 10431053, 2004.

WEILL, M. A. M.; SPAROVEK, G. Estudo da erosão na microbacia do Ceveiro (Piracicaba, SP). I - Estimativa das taxas de perda de solo e estudo de sensibilidade dos fatores do modelo EUPS. Revista Brasileira de Ciência do Solo, v. 32, p. 801-814, 2008.

WISCHMEIER, W. H.; SMITH, D. D. Predicting rainfall erosion losses. A guide to conservation planning. Washington: USDA Agriculture Handbook, 1978. 